\title{
ON SEQUENTIALLY COHEN-MACAULAY COMPLEXES AND POSETS
}

\author{
ANDERS BJÖRNER, MICHELLE WACHS ${ }^{1}$, AND VOLKMAR WELKER ${ }^{2}$
}

\begin{abstract}
The classes of sequentially Cohen-Macaulay and sequentially homotopy Cohen-Macaulay complexes and posets are studied. First, some different versions of the definitions are discussed and the homotopy type is determined. Second, it is shown how various constructions, such as join, product and rank-selection preserve these properties. Third, a characterization of sequential Cohen-Macaulayness for posets is given. Finally, in an appendix we outline connections with ring-theory and survey some uses of sequential Cohen-Macaulayness in commutative algebra.
\end{abstract}

\section{INTRODUCTION}

The notion of sequential Cohen-Macaulayness is a nonpure generalization, due to Stanley [17, Sec. III.2], of the notion of CohenMacaulayness. Stanley introduced this in order to provide a ringtheoretic complement to the theory of nonpure shellability [4, 5]. Just as pure shellability implies Cohen-Macaulayness, nonpure shellability implies sequential Cohen-Macaulayness.

In this paper we show that the most common Cohen-Macaulay preserving constructions on simplicial complexes and posets also preserve sequential Cohen-Macaulayness. This complements earlier results [4, 5] showing that these operations preserve nonpure shellability.

We also discuss a nonpure version of Quillen's concept [15] of homotopy Cohen-Macaulayness, introduced by the authors in [6]. This new concept is intermediate between nonpure shellability and sequential Cohen-Macaulayness. We show that the homotopy version of sequential Cohen-Macaulayness has the same strong topological consequences as that of (nonpure) shellability, namely having the homotopy type of a wedge of spheres of (possibly) varying dimensions.

Date: October 30, 2018.

1. Supported in part by National Science Foundation grants DMS DMS 0302310 and DMS 0604562.

2. Supported by Deutsche Forschungsgemeinschaft (DFG). 
Our primary goal is to extend results on Cohen-Macaulay simplicial complexes, that have proven to be useful, to sequentially CohenMacaulay simplicial complexes. Some of the proofs are straightforward generalizations of the Cohen-Macaulay versions, while others require substantially new ideas. For basic facts from topological combinatorics we refer to the survey article by Björner [3] and for background in commutative algebra to the books by Stanley [17] and Bruns \& Herzog [8].

As was mentioned, the notion of sequential Cohen-Macaulayness was first defined in terms of commutative algebra by Stanley. In [17] he also gave a homological characterization, see Appendix II, where this is outlined. Starting from Stanley's homological characterization, two other homological characterizations were found by Duval [10] and Wachs [18. We take Wachs' characterization as our definition, and we will return to Duval's in the next section (see Proposition 2.5).

Let $\Delta$ be a simplicial complex, and for $0 \leq m \leq \operatorname{dim} \Delta$, let $\Delta^{\langle m\rangle}$ be the subcomplex of $\Delta$ generated by its facets of dimension $\geq m$.

\section{Definition 1.1.}

(i) The complex $\Delta$ is sequentially acyclic over $\mathbf{k}$ if $\Delta^{\langle m\rangle}$ is $(m-1)$ acyclic over $\mathbf{k}$ for all $m=0,1, \ldots, \operatorname{dim} \Delta$, i.e., $\tilde{H}_{r}\left(\Delta^{\langle m\rangle} ; \mathbf{k}\right)=0$ for all $r<m \leq \operatorname{dim} \Delta$, where $\mathbf{k}$ is the ring of integers or a field.

(ii) The complex $\Delta$ is sequentially connected if $\Delta^{\langle m\rangle}$ is $(m-1)$ connected for all $m=0,1, \ldots, \operatorname{dim} \Delta$, i.e., $\pi_{r}\left(\Delta^{\langle m\rangle}\right)=0$ for all $r<m \leq \operatorname{dim} \Delta$.

Recall that the link of a face $F$ in $\Delta$ is defined to be the subcomplex

$$
\mathrm{lk}_{\Delta} F=\{G \in \Delta \mid F \cup G \in \Delta, F \cap G=\emptyset\} \text {. }
$$

\section{Definition 1.2.}

(i) The complex $\Delta$ is sequentially Cohen-Macaulay over $\mathbf{k}$ if $\operatorname{lk}_{\Delta} F$ is sequentially acyclic over $\mathbf{k}$ for all $F \in \Delta$.

(Usually we will drop the reference to $\mathbf{k}$ and just say "sequentially acyclic" and "sequentially Cohen-Macaulay" (SCM)).

(ii) $\Delta$ is sequentially homotopy Cohen-Macaulay (SHCM) if $\mathrm{lk}_{\Delta} F$ is sequentially connected for all $F \in \Delta$.

A simplicial complex is said to be pure if all its facets are of equal dimension. Clearly, a pure $d$-dimensional simplicial complex is sequentially connected if and only if it is $(d-1)$-connected, and it is sequentially acyclic if and only if it is $(d-1)$-acyclic. It follows that for pure simplicial complexes, the notion of "S(H)CM" reduces to the notion of "(homotopy) Cohen-Macaulay". 
A poset $P$ is said to be pure, sequentially connected, sequentially acyclic, SCM, or SHCM if its order complex $\Delta(P)$ is, where $\Delta(P)$ is the simplicial complex of chains of $P$.

The paper is organized as follows. In Section 2, we show that a SHCM complex has the homotopy type of a wedge of spheres in the dimensions of the facets of the complex. The homology version of this result appears in [18.

The main result of Section 3 is that sequential connectivity, sequential acyclicity, SHCMness, and SCMness are all preserved by taking joins. The proof of this in the pure case is quite simple, but in the nonpure case relies on a fiber lemma of Quillen. There are several interesting poset consequences of this result, in particular a nice characterization of $\mathrm{S}(\mathrm{H}) \mathrm{CM}$ posets, and the result that $\mathrm{S}(\mathrm{H})$ CMness is preserved by taking products of posets with minimum elements.

In Section 4 we prove some general results on induced subcomplexes of $\mathrm{S}(\mathrm{H}) \mathrm{CM}$ complexes that enable us to show that rank-selection on semipure posets preserves the $\mathrm{S}(\mathrm{H}) \mathrm{CM}$ property and that truncation on general posets also preserves the $\mathrm{S}(\mathrm{H}) \mathrm{CM}$ property.

Section 5 contains a poset analog of Duval's characterization of SCMness, which does not follow directly from Duval's simplicial complex characterization. This leads to a characterization of SCMness of semipure posets in terms of rank-selection, which extends a result in the pure case due to Baclawski and Garsia [1] and Walker [20].

Since Walker's rank-selection result is unpublished and one of our results relies on this, we present his proof in an appendix. In another appendix we outline the connection with commutative algebra. We give Stanley's definition of sequentially Cohen-Macaulay modules and sketch the connection to the concept of sequentially Cohen-Macaulay simplicial complexes as defined in this paper. In this appendix we also survey some of the uses that the concept of sequential CohenMacaulayness has found in the recent research literature.

\section{Sequentially COnNeCted COMplexes}

We begin with a description of the homotopy type of a sequentially connected complex. The corresponding homological fact for sequentially acyclic complexes is known from [18].

Proposition 2.1 ([18, Proposition 1.9]). Let $\Delta$ be sequentially acyclic over $\mathbf{k}$. Then $\tilde{H}_{*}(\Delta ; \mathbf{k})$ is free and $\tilde{H}_{i}(\Delta ; \mathbf{k})=0$ if $\Delta$ has no facet of dimension $i$. 
Theorem 2.2. Suppose that $\Delta$ is a simplicial complex with facets of dimensions $d_{1}, \ldots, d_{t}$. If $\Delta$ is sequentially connected, then it has the homotopy type of a wedge of spheres of dimensions in $\left\{d_{1}, \ldots, d_{t}\right\}$.

Proof. Assume that $d_{1}>\cdots>d_{t}$. We start with the case that $d_{t} \geq$ 2 , so that $\Delta=\Delta^{\left\langle d_{t}\right\rangle}$ is simply-connected. We already know from Proposition 2.1 that $\tilde{H}_{*}(\Delta ; \mathbb{Z})$ and $\tilde{H}_{*}\left(\Delta^{\langle i\rangle} ; \mathbb{Z}\right)$ are free for all $i$. Let $\beta_{i}=$ $\operatorname{rank} \tilde{H}_{i}(\Delta ; \mathbb{Z})=\operatorname{rank} \tilde{H}_{i}\left(\Delta^{\langle i\rangle} ; \mathbb{Z}\right)$. We also know from Proposition 2.1 that $\beta_{i}=0$ for all $i<2$.

Since $\Delta^{\langle i\rangle}$ is $(i-1)$-connected, the Hurewicz theorem [7, p. 479] gives the existence of an isomorphism $h_{i}: \pi_{i}\left(\Delta^{\langle i\rangle}\right) \rightarrow \tilde{H}_{i}\left(\Delta^{\langle i\rangle} ; \mathbb{Z}\right)$ when $i \geq 2$. This means that we can find mappings $\varphi_{j}^{i}\left(j=1, \ldots, \beta_{i}\right)$ from the $i$-sphere to $\Delta^{\langle i\rangle}$ whose induced homology classes form a basis for the free group $\tilde{H}_{i}\left(\Delta^{\langle i\rangle} ; \mathbb{Z}\right)$. Let $W$ be a wedge of spheres having $\beta_{i}$ $i$-dimensional spheres for all $i$. Since $\tilde{H}_{i}(\Delta ; \mathbb{Z})=\tilde{H}_{i}\left(\Delta^{\langle i\rangle} ; \mathbb{Z}\right)$ we can piece the mappings $\varphi_{j}^{i}$ together to a single mapping $\Phi: W \rightarrow \Delta$ which induces isomorphism of homology in all dimensions. Since $W$ and $\Delta$ are simply-connected, the Whitehead theorem [7, p. 486] implies that such a mapping is a homotopy equivalence $\Phi: W \simeq \Delta$.

Assume now that $d_{t}=1$, and write $\Delta=\Delta^{\langle 2\rangle} \cup \Gamma$, where $\Gamma$ is the 1-skeleton of $\Delta$ (which is assumed to be connected). Note that $\Delta^{\langle 2\rangle}$ (assumed to be simply-connected) is by the preceding homotopy equivalent to a wedge of spheres. Let $T^{\prime}$ be a spanning-tree of the 1-skeleton of $\Delta^{\langle 2\rangle}$, and extend $T^{\prime}$ to a spanning tree $T$ of $\Gamma$. Collapsing the tree $T$ turns $\Delta$ into a wedge of the space $\Delta^{\langle 2\rangle} / T^{\prime}$ with a collection of loops (1-spheres), one coming from each edge in $\Gamma \backslash T$. Now, collapsing a contractible subspace does not change homotopy type [7, p. 436], so

$$
\Delta \simeq \Delta / T \simeq\left(\Delta^{\langle 2\rangle} / T^{\prime}\right) \bigvee\{\text { loops }\} \simeq \Delta^{\langle 2\rangle} \bigvee\{\text { loops }\}
$$

In the $d_{t}=0$ case there are in addition to the previous situation only some isolated vertices. To handle these requires only adding the corresponding number of 0 -spheres to the wedge already constructed.

It was observed by Stanley [17, p. 87] that, just as in the pure case, SCMness is a topological property, i.e., a property that depends only on the geometric realization of the simplicial complex and $\mathbf{k}$; see [19, Theorem 4.1.6]. Although this is not true for the homotopy version (see [15, Section 8]), it is easily seen to be true for both sequential connectivity and sequential acyclicity.

Proposition 2.3. Sequential connectivity and sequential acyclicity are topological properties. 
Proof. Given a nonnegative integer $m$ and a topological space $X$, define $X^{\langle m\rangle}$ to be the topological closure of the set

$$
\begin{gathered}
\{p \in X: p \text { has a neighborhood homeomorphic to } \\
\text { an open } d \text {-ball where } d \geq m\} .
\end{gathered}
$$

The result follows from the fact that $\left\|\Delta^{\langle m\rangle}\right\|=\|\Delta\|^{\langle m\rangle}$, for all $m=$ $0,1, \ldots, \operatorname{dim} \Delta$, where $\|\Delta\|$ denotes the geometric realization of $\Delta$.

There is a characterization of SCMness due to Duval [10] which involves the pure $r$-skeleton of a simplicial complex. The pure r-skeleton $\Delta^{[r]}$ of a simplicial complex $\Delta$ is defined to be the subcomplex of $\Delta$ generated by all faces of dimension $r$. In Proposition 2.5 below we give Duval's formulation and a homotopy version of it. It is shown in [18, Theorem 1.5] that Duval's formulation is equivalent to the one used here (Definition 1.2), by observing the following connection between homology of the pure $r$-skeleton and sequential acyclicity. Recall that a simplicial complex $\Delta$ is said to be spherical if it is $(\operatorname{dim}(\Delta)-1)$ connected. We say that $\Delta$ is homology-spherical if it is $(\operatorname{dim}(\Delta)-1)$ acyclic.

Lemma 2.4. A simplicial complex $\Delta$ is sequentially connected (acyclic) if and only if its pure $r$-skeleton $\Delta^{[r]}$ is (homology-)spherical for all $r \leq \operatorname{dim} \Delta$.

Proof. Let $\Delta^{r}$ denote the $r$-skeleton of $\Delta$. Since $\Delta^{[r]}=\left(\Delta^{<r>}\right)^{r}$, we have $\pi_{i}\left(\Delta^{[r]}\right)=\pi_{i}\left(\Delta^{<r>}\right)$ for all $i<r$. Hence $\Delta^{[r]}$ is $(r-1)$-connected if and only if $\Delta^{<r>}$ is. It follows that $\Delta$ is sequentially connected if and only if all the pure skeleta of $\Delta$ are spherical.

An analogous argument works for homology.

The homology version of the following result appears in [18, Theorem $1.5]$.

Proposition 2.5. A simplicial complex $\Delta$ is $S(H) C M$ if and only if its pure r-skeleton $\Delta^{[r]}$ is (H)CM for all $r \leq \operatorname{dim} \Delta$.

Proof. It is easy to see that if $F \in \Delta^{[r]}$ then

$$
\operatorname{lk}_{\Delta}(F)^{[r-\operatorname{dim} F-1]}=\mathrm{lk}_{\Delta^{[r]}}(F) .
$$

It therefore follows from Lemma 2.4 that $\operatorname{lk}_{\Delta^{[r]}}(F)$ is spherical for all $r$ such that $F \in \Delta^{[r]}$ if and only if $\operatorname{lk}_{\Delta}(F)$ is sequentially connected. This means that $\Delta^{[r]}$ is HCM for all $r$ if and only if $\operatorname{lk}_{\Delta}(F)$ is sequentially connected for all $F$.

The analogous argument works for homology [18].

For more about the definition of sequential Cohen-Macaulayness, see Appendix II. 


\section{JOIN AND PRODUCT}

This section and the next deal with constructions on complexes and posets that preserve SHCMness, SCMness, sequential connectivity, and sequential acyclicity.

Let $P$ be a finite poset, and for $x \in P$ let $P_{\leq x}$ denote the principal order ideal $\{y \in P \mid y \leq x\}$ and let $P_{<x}$ denote $\{y \in P \mid y<x\}$. For $x \leq y \in P$, let $[x, y]$ denote the closed interval $\{z \in P \mid x \leq z \leq y\}$ and let $(x, y)$ denote the open interval $\{z \in P \mid x<z<y\}$.

The following "Quillen fiber lemma" is the main tool in all of the results to follow.

Lemma 3.1. 15, Proposition 7.6]. Let $f: P \rightarrow Q$ be a poset map, and suppose that the fiber $\Delta\left(f^{-1}\left(Q_{\leq q}\right)\right)$ is t-connected for all $q \in Q$. Then $\Delta(P)$ is t-connected if and only if $\Delta(Q)$ is t-connected.

The same is true with " $t$-connected" everywhere replaced by " $t$ acyclic".

The following result is in the pure case an immediate consequence of the fact that the join of an $r$-connected complex and an $s$-connected complex is $(r+s-2)$-connected. Its proof in the nonpure case relies on Lemma 3.1. It is used to prove the remaining results of this section.

Theorem 3.2. The join of two sequentially connected (acyclic) simplicial complexes is sequentially connected (acyclic).

Proof. Suppose $\Delta$ and $\Gamma$ are sequentially connected. We will show that $(\Delta * \Gamma)^{\langle m\rangle}$ is $(m-1)$-connected for all $m$ by using Lemma 3.1. Let $P$ be the poset of nonempty faces of $(\Delta * \Gamma)^{\langle m\rangle}$ and let $Q$ be the poset of nonempty closed intervals of the totally ordered set

$$
(m-\operatorname{dim} \Gamma-1)<(m-\operatorname{dim} \Gamma)<\cdots<\operatorname{dim} \Delta,
$$

ordered by reverse inclusion. We construct a poset map from $P$ to $Q$. Let $F \in(\Delta * \Gamma)^{\langle m\rangle}$. Then $F=F_{1} \cup F_{2}$ where $F_{1} \in \Delta$ and $F_{2} \in \Gamma$. Let $r=\max \left\{\operatorname{dim} G \mid F_{1} \subseteq G \in \Delta\right\}$ and $s=\max \left\{\operatorname{dim} G \mid F_{2} \subseteq G \in \Gamma\right\}$. Define $f: P \rightarrow Q$ by $f(F)=[m-r-1, s]$. It is easy to see that $f$ is order preserving, and that for each $[a, b] \in Q$, the fiber $f^{-1}\left(Q_{\leq[a, b]}\right)$ is the poset of nonempty faces of $\Delta^{\langle m-a-1\rangle} * \Gamma^{\langle b\rangle}$. It follows from the assumption that $\Delta$ and $\Gamma$ are sequentially connected that $\Delta^{\langle m-a-1\rangle}$ is $(m-a-2)$-connected and $\Gamma^{\langle b\rangle}$ is $(b-1)$-connected. Since the join of an $r$-connected complex and an $s$-connected complex is $(r+s+2)$ connected, it follows that the order complex of the fiber $f^{-1}\left(Q_{\leq[a, b]}\right)$ is $(m-a-2+b-1+2)$-connected. Since $m-a-2+b-1+2 \geq$ $m-1$, the order complex of each fiber is $(m-1)$-connected. Also $\Delta(Q)$ is contractible since $Q$ has a minimum element. Hence, Lemma 
3.1 implies that $\Delta(P)$ is $(m-1)$-connected, which in turn implies that $(\Delta * \Gamma)^{\langle m\rangle}$ is $(m-1)$-connected.

The homology version of the result is proved analogously.

Corollary 3.3. The join of simplicial complexes $\Delta$ and $\Gamma$ is $S(H) C M$ if and only if $\Delta$ and $\Gamma$ are $S(H) C M$.

Proof. Use the fact that the link of a face in $\Delta * \Gamma$ is the join of the links of faces in $\Delta$ and $\Gamma$.

Recall that the ordinal sum of two posets $P$ and $Q$ is the poset on the disjoint union of $P$ and $Q$, whose order relation restricts to the ones on $P$ and $Q$ and sets all $p \in P$ below all $q \in Q$.

Corollary 3.4. The ordinal sum of posets is sequentially connected (sequentially acyclic, SHCM, SCM) if and only if each poset is.

Since the links of faces of the order complex of a poset $P$ are the order complexes of ordinal sums of open intervals of $\hat{P}$, where $\hat{P}$ is the poset $P$ with a minimum element $\hat{0}$ and a maximum element $\hat{1}$ attached, we have the following nice characterization of $\mathrm{S}(\mathrm{H}) \mathrm{CM}$ posets.

Corollary 3.5. A poset $P$ is $S(H) C M$ if and only if every open interval of $\hat{P}$ is sequentially (connected) acyclic.

Given a simplicial complex $\Delta$, let $P(\Delta)$ denote the poset of nonempty faces of $\Delta$ ordered by inclusion. Since $\Delta(P(\Delta))$ is the barycentric subdivision of $\Delta$, the two complexes are homeomorphic. We thus have the following consequence of Proposition 2.3 and Corollary 3.5 .

Corollary 3.6. A simplicial complex $\Delta$ is sequentially connected (sequentially acyclic, SHCM, SCM) if and only if $P(\Delta)$ is sequentially connected (sequentially acyclic, SHCM, SCM).

Proof. For sequential connectivity and acyclicity, this is an immediate consequence of the fact that they are topological properties (Proposition 2.3). For SCMness it is also an immediate consequence of the fact that SCMness is a topological property, which is more difficult to prove than Proposition 2.3. However, the result for both SCMness and SHCMness can be shown to follow from the respective results for sequentially connectivity and sequential acyclicity and from Corollary 3.5. Indeed, observe that $P\left(\mathrm{lk}_{\Delta} F\right)$ is isomorphic to the principal upper order ideal $\{G \in \Delta \mid F \subsetneq G\}$ of $P(\Delta)$. Hence $\Delta$ is SHCM if and only if every open principal upper order ideal of $P(\Delta)$ is sequentially connected. Each of the other open intervals of $P(\Delta) \cup\{\hat{0}, \hat{1}\}$ is isomorphic to the proper part of a Boolean algebra, and hence is sequentially 
connected no matter what $\Delta$ is. Consequently $\Delta$ is SHCM if and only if every open interval of $P(\Delta) \cup\{\hat{0}, \hat{1}\}$ is sequentially connected. By Corollary 3.5, $\Delta$ is SHCM if and only if $P(\Delta)$ is.

We now turn to the poset product operation. If $P_{i}$ is a finite poset, then let $\dot{P}_{i}$ be the poset $P_{i}$ with a minimum element $\hat{0}_{i}$ attached, and $\hat{P}_{i}$ be the poset $P_{i}$ with a minimum element $\hat{0}_{i}$ and a maximum element $\hat{1}_{i}$ attached.

Corollary 3.7. Let $P_{1}$ and $P_{2}$ be sequentially (connected) acyclic posets. Then

(1) $\dot{P}_{1} \times \dot{P}_{2} \backslash\left\{\left(\hat{0}_{1}, \hat{0}_{2}\right)\right\}$ is sequentially (connected) acyclic,

(2) $\hat{P}_{1} \times \hat{P}_{2} \backslash\left\{\left(\hat{0}_{1}, \hat{O}_{2}\right),\left(\hat{1}_{1}, \hat{1}_{2}\right)\right\}$ is sequentially (connected) acyclic.

Proof. By a result of Quillen [15] (see [21, Theorem 5.1 (b)]), there is a homeomorphism from $\Delta\left(\dot{P}_{1} \times \dot{P}_{2} \backslash\left\{\left(\hat{0}_{1}, \hat{0}_{2}\right)\right\}\right)$ onto $\Delta\left(P_{1}\right) * \Delta\left(P_{2}\right)$. Hence (1) follows from Theorems 2.3 and 3.2 . Similarly, a homeomorphism of Walker [21, Theorem 5.1 (d)] yields (2).

Corollary 3.8. Let $P_{1}$ and $P_{2}$ be posets with minimum elements $\hat{0}_{1}$ and $\hat{0}_{2}$, respectively. Then $P_{1} \times P_{2}$ is $S(H) C M$ if and only if $P_{1}$ and $P_{2}$ are $S(H) C M$.

Proof. Suppose $P_{1}$ and $P_{2}$ are SHCM. Then $P_{i} \backslash\left\{\hat{0}_{i}\right\}$ is SHCM. By applying Corollary 3.7 (1) to $P_{i} \backslash\left\{\hat{0}_{i}\right\}$ we get that $P_{1} \times P_{2} \backslash\left\{0_{1}, 0_{2}\right\}$ is sequentially connected, which implies that $P_{1} \times P_{2}$ is sequentially connected. That all upper order ideals $\left(P_{1} \times P_{2}\right)_{>\left(x_{1}, x_{2}\right)}$ are sequentially connected also follows from from Corollary 3.7 (1), and that all open intervals $\left(\left(x_{1}, x_{2}\right),\left(y_{1}, y_{2}\right)\right)$ of $P_{1} \times P_{2}$ are sequentially connected follows from Corollary 3.7 (2). Now by Corollary 3.5 $P_{1} \times P_{2}$ is SHCM. Conversely, if $P_{1} \times P_{2}$ is SHCM then the open interval $\left(P_{1} \times P_{2}\right)_{>\left(x, \hat{0}_{2}\right)}$, where $x$ is maximal in $P_{1}$, is SHCM. Since this open interval is isomorphic to $P_{2} \backslash\left\{\hat{0}_{2}\right\}$, we conclude that $P_{2}$ is SHCM. Similarly $P_{1}$ is SHCM.

An analogous argument yields the homology version.

The interval poset $\operatorname{Int}(P)$ of a poset $P$ is the poset of closed intervals of $P$ ordered by inclusion.

Corollary 3.9. A poset $P$ is $S(H) C M$ if and only if $\operatorname{Int}(P)$ is $S(H) C M$.

Proof. The proof, which is similar to that of Corollary 3.8, uses the homeomorphism in [21, Theorem 6.1]. 


\section{RANK SELECTION}

Throughout this section, we assume that $P$ is a poset with a minimum element $\hat{0}$. The length $\ell(P)$ of $P$ is the length of the longest chain of $P$, where the length of a chain is its cardinality minus one. For $x \in P$, define the rank,

$$
r(x):=\ell([\hat{0}, x]) .
$$

For $S \subseteq\{0,1, \ldots, \ell(P)\}$, define the rank-selected subposet

$$
P_{S}:=\{x \in P \mid r(x) \in S\} .
$$

It is well-known that rank-selection preserves the Cohen-Macaulay property in the pure case, see e.g. [1] and [3, p. 1858] for references. We shall show that the same is true in the semipure case, where $P$ is said to be semipure if $[\hat{0}, x]$ is pure for all $x \in P$. For general bounded posets this is true only for special types of rank-selection.

We begin with two lemmas. The proof of the first lemma, given in [3], relies on Lemma 3.1. It is used to prove the second lemma, which is key to the rest of this section.

Given a simplicial complex $\Delta$ and a subset $A$ of its vertex set, the induced subcomplex $\Delta(A)$ is defined by

$$
\Delta(A):=\{F \in \Delta \mid F \subseteq A\} .
$$

Lemma 4.1 ([3, Lemma 11.11]). Let $\Delta$ be a simplicial complex on vertex set $V$, and let $A \subseteq V$. Assume that $\operatorname{lk}_{\Delta}(F)$ is m-connected for all $F \in \Delta(V \backslash A)$. Then $\Delta(A)$ is m-connected if and only if $\Delta$ is $m$-connected.

The same is true with " $m$-connected" everywhere replaced by " $m$ acyclic".

Lemma 4.2. Let $\Delta$ be a $S(H) C M$ simplicial complex on vertex set $V$. Let $A \subseteq V$ be such that for all facets $F \in \Delta$,

$$
|(V \backslash A) \cap F|= \begin{cases}1 & \text { if } \operatorname{dim} F \geq t \\ 0 & \text { otherwise }\end{cases}
$$

where $t$ is some fixed element of $\{0,1, \ldots, \operatorname{dim} \Delta\}$. Then the induced subcomplex $\Delta(A)$ is $S(H) C M$.

Proof. We give the proof for the homotopy version. An analogous argument yields the homology version. The proof is based on that of a pure version given in [3, Theorem 11.13].

We begin by using Lemma 4.1 to show that $\Delta(A)^{\langle m\rangle}$ is $(m-1)$ connected for all $m=0,1, \ldots, \operatorname{dim} \Delta(A)$. First note that since $\Delta_{V \backslash A}$ 
is 0-dimensional, we only need to consider links of vertices that are not in $A$ when applying Lemma 4.1 .

Case 1. $m<t$. In this case

$$
\Delta(A)^{\langle m\rangle}=\Delta^{\langle m\rangle}(A) .
$$

Let $x \in V \backslash A$. Since no facet of dimension less than $t$ contains $x$, we have

$$
\begin{aligned}
& \operatorname{lk}_{\Delta\langle m\rangle}(x)=1 \mathrm{k}_{\Delta}\langle t\rangle(x) \\
& =\left(\mathrm{lk}_{\Delta} x\right)^{\langle t-1\rangle} \text {. }
\end{aligned}
$$

Since $\mathrm{lk}_{\Delta} x$ is sequentially connected, we have that $\mathrm{lk}_{\Delta}\langle m\rangle(x)$ is $(t-2)$ connected, which implies it is $(m-1)$-connected. Since $\Delta$ is sequentially connected, $\Delta^{\langle m\rangle}$ is also $(m-1)$-connected. Hence, by Lemma 4.1, $\Delta^{\langle m\rangle}(A)$ is $(m-1)$-connected. By (1), $\Delta(A)^{\langle m\rangle}$ is $(m-1)$-connected.

Case 2. $m \geq t$. In this case

$$
\Delta(A)^{\langle m\rangle}=\Delta^{\langle m+1\rangle}(A) .
$$

Let $x \in V \backslash A$. We have $\operatorname{lk}_{\Delta\langle m+1\rangle}(x)=\left(\operatorname{lk}_{\Delta} x\right)^{\langle m\rangle}$, which is $(m-1)$ connected since $\operatorname{lk}_{\Delta} x$ is sequentially connected. Also $\Delta^{\langle m+1\rangle}$ is $(m-$ 1 )-connected for the same reason. Hence by Lemma 4.1, $\Delta^{\langle m+1\rangle}(A)$ is $(m-1)$-connected, which means by (2) that $\Delta(A)^{\langle m\rangle}$ is $(m-1)$ connected.

We can now conclude that $\Delta(A)$ is sequentially connected. To complete the proof we need to show that all links of $\Delta(A)$ are sequentially connected. This follows from the fact that for all $F \in \Delta(A)$,

$$
\mathrm{lk}_{\Delta(A)} F=\left(\mathrm{lk}_{\Delta} F\right)(A) .
$$

Indeed, since the hypothesis of the lemma holds for the sequentially Cohen-Macaulay complex $\operatorname{lk}_{\Delta} F$ and the set $A$, the above argument yields the conclusion that $\left(\mathrm{lk}_{\Delta} F\right)(A)$ is sequentially connected.

A completely balanced simplicial complex is defined to be a simplicial complex $\Delta$ together with a "coloring" function $\tau: V \rightarrow \mathbb{N}$ such that for each facet $F$, we have $\tau(F)=\{0,1,2, \ldots,|F|-1\}$. The order complex of a semipure poset with rank function serving as color function is the prototypical example a completely balanced simplicial complex.

Theorem 4.3. Let $\Delta$ be a completely balanced d-dimensional $S(H) C M$ simplicial complex. Then for all $S \subseteq\{0,1, \ldots, d\}$, the type-selected subcomplex $\Delta_{S}:=\{G \in \Delta \mid \tau(G) \subseteq S\}$ is $S(H) C M$.

Proof. By induction, we can assume that $|S|=d$. Let $t$ be the unique element of $\{0,1, \ldots, d\}-S$. Let $A$ be the set of vertices whose color is not $t$. Since $\Delta$ is completely balanced, each facet of dimension at least 
$t$ contains exactly one vertex of color $t$ (i.e., exactly one vertex in the complement of $A$ ) and each facet of dimension less than $t$ contains no vertices of color $t$. The result now follows from Lemma 4.2.

In the case of posets, the theorem reduces to the following:

Corollary 4.4. Let $P$ be a semipure $S(H) C M$ poset of length $\ell$. For all $S \subseteq\{0,1, \ldots, \ell\}$, the rank-selected subposet $P_{S}$ is $S(H) C M$.

Theorem 4.5. Let $P$ be a semipure $S(H) C M$ poset of length $\ell$. For all $t=0,1, \ldots, \ell$, the max-deleted subposet

$$
P^{(t)}:=P-\{x \in P \mid x \text { is a maximal element and } r(x) \geq t\}
$$

is $S(H) C M$.

Proof. Apply Lemma 4.2 to the order complex of $P$ by setting $A=$ $P^{(t)}$.

Corollary 4.6. Let $\Delta$ be a $S(H) C M$ simplicial complex. Then the $t$-skeleton and $t$-coskeleton of $\Delta$ are $S(H) C M$ for all $t$.

Proof. The $t$-skeleton is obtained by rank-selection and the $t$-coskeleton is obtained by repeated max-deletion in the face poset of $\Delta$.

Now let us consider rank-selection in general posets. Let $P$ be a bounded poset, i.e., a poset with a minimum element and a maximum element. The corank $r^{*}(x)$ of $x \in P$ is the rank of $x$ in the dual poset $P^{*}$. Note that when $P$ is pure, $r^{*}(x)=\ell(P)-r(x)$. For $S, T \subseteq$ $\{0,1, \ldots, \ell(P)\}$, define the rank selected subposet

$$
P_{S}^{T}:=\left\{x \in P \mid r(x) \in S \text { and } r^{*}(x) \in T\right\} .
$$

Rank selection in the general setting does not preserve $\mathrm{S}(\mathrm{H})$ CMness, see [4, Figure 9a]. However, a special type of rank-selection, called truncation, does preserve $\mathrm{S}(\mathrm{H}) \mathrm{CMness}$, as can be seen in the next result.

Theorem 4.7. Let $P$ be a bounded $S(H) C M$ poset of length $\ell$. For $0 \leq s, t<\ell$, let $S=\{s, s+1, \ldots, \ell\}$ and $T=\{t, t+1, \ldots, \ell\}$. Then the truncation $P_{S}^{T}$ is $S(H) C M$.

Proof. Since $P_{S}^{T}=\left(\left(\left(P_{S}\right)^{*}\right)_{T}\right)^{*}$, we need only prove the result for $P_{S}$. Assume $s \geq 2$ (the result is trivial otherwise). The poset $P_{S}$ can be obtained by first removing $\hat{0}$ and then repeatedly removing all the atoms. Apply Lemma 4.2 to the order complex of $P \backslash\{\hat{0}\}$ with $A=$ $(P \backslash\{\hat{0}\}) \backslash\{$ atoms $\}$. 


\section{A poset analog of Duval's Characterization}

For a semipure poset $P$, let $P^{<j>}$ be the lower order ideal generated by all maximal elements of rank at least $j$ and let $P^{[j]}$ be the lower order ideal generated by all elements of rank $j$. Clearly $\Delta\left(P^{<j>}\right)=\Delta(P)^{<j>}$. Hence one has the following poset version of Definition 1.1: A semipure poset $P$ is is sequentially (connected) acyclic if and only if $P^{<j>}$ is $(j-1)$-acyclic (-connected), for all $j=0, \ldots, \ell(P)$. By combining this with Corollary 3.5, one gets a nice characterization of $\mathrm{S}(\mathrm{H}) \mathrm{CMness}$ for semipure posets.

It is clearly not the case that $\Delta\left(P^{[j]}\right)=\Delta(P)^{[j]}$. Hence a poset version of Duval's characterization of SCM (Proposition 2.5) does not follow directly from his characterization for simplicial complexes. Nevertheless a poset version of Duval's characterization does indeed hold.

Theorem 5.1. A semipure poset $P$ is $S C M$ (resp. SHCM) if and only if $P^{[j]}$ is $C M$ (resp. HCM) for all $j$.

Proof. Suppose $P$ is $\mathrm{S}(\mathrm{H}) \mathrm{CM}$. Then $P^{<j>}$ is sequentially (connected) acyclic for all $j$. All intervals of $P^{<j>}$ are intervals of $P$ and all principal upper order ideals of $P^{<j>}$ have the form $I^{<k>}$, where $I$ is a principal upper order ideal of $P$ and $k$ is some integer. Hence by Corollary 3.5, all intervals of $\widehat{P<j>}$ are sequentially (connected) acyclic and therefore $P^{<j>}$ is $\mathrm{S}(\mathrm{H}) \mathrm{CM}$. It follows from Corollary 4.4 that $P^{[j]}$ is $(\mathrm{H}) \mathrm{CM}$ for all $j$ since $P^{[j]}=P_{\{0,1, \ldots, j\}}^{<j>}$.

We now prove the homotopy version of the converse and leave the analogous proof of the homology version to the reader. Suppose $P$ semipure and $P^{[j]}$ is HCM for all $j$. We will use Corollary 3.5 to show that $P$ is SHCM. First we establish sequential connectivity for $P$. We show that $P^{<j>}$ is $(j-1)$-connected for all $j$ by induction on $\ell(P)-j$. If $j=\ell(P)$ then $P^{<j>}=P^{[j]}$, which is $(j-1)$-connected. Now assume $j<\ell(P)$. If $P$ has no maximum element of rank $j$ then $P^{<j>}=P^{<j+1>}$, which by induction is $j$-connected and hence $(j-1)$ connected.

Now assume $P$ has a maximal element of rank $j$. Then $P^{<j>}=$ $P^{<j+1>} \cup P^{[j]}$. By induction, $P^{<j+1>}$ is $j$-connected and hence $(j-$ $1)$-connected. Also $P^{[j]}$ is $(j-1)$-connected since it is HCM. The intersection $P^{<j+1>} \cap P^{[j]}$ is the rank-selected subposet $\left(P^{[j+1]}\right)_{\{0,1, \ldots, j\}}$, which is HCM (by Theorem 4.4) and therefore $(j-1)$-connected. By the Meyer-Vietoris Theorem [7, p. 229], $P^{<j>}$ is $(j-1)$-acyclic for all $j$, and by the Seifert - Van Kampen Theorem [7, p. 161], $P^{<j>}$ is simply connected. It therefore follows from the Hurewicz Theorem [7, p. 479] that $P^{<j>}$ is $(j-1)$-connected. We can now conclude that $P$ 
is sequentially connected whenever $P$ is a semipure poset for which all the $P^{[j]}$ are HCM.

Next we establish sequential connectivity for all intervals and principal upper order ideals of $P$. Intervals of $P$ are intervals of some $P^{[j]}$, so they are indeed sequentially connected. Let $I$ be a principal upper order ideal of $P$. For each $j=0, \ldots, \ell(I)$, the pure poset $I^{[j]}$ is a principal upper order ideal of $P^{[k]}$ where $k-j$ is the rank (in $P$ ) of the minimum element of $I$. Since $P^{[k]}$ is HCM, so is the upper order ideal $I^{[j]}$. Since $I$ is a semipure poset for which all $I^{[j]}$ are HCM, by the result of the previous paragraph, we conclude that $I$ is sequentially connected. It now follows from Corollary 3.5 that $P$ is SHCM.

It is known from work of Baclawski and Garsia [1] that CohenMacaulay posets are characterized by the property that all rank-selected subposets are homology-spherical. A sharpening of this result and of its homotopy version was given by James W. Walker [20]. The sharpening consists in the observation that it suffices to consider intervals of ranks. By an interval of ranks $S$ of a pure poset $P$ we mean a set of consecutive ranks $S=\{a, a+1, a+2, \ldots\} \subseteq\{1,2, \ldots, \ell(P \cup\{\hat{0}\})\}$. If $P$ lacks a minimum element then by $P_{S}$ we mean $(P \cup\{\hat{0}\})_{S}$. (Recall rank-selection was defined in Section 4 only for posets that have a minimum element.)

Theorem 5.2 (Walker [20]). If $P$ is a pure poset such that the rankselected subposet $P_{S}$ is spherical (resp. homology-spherical) for every interval of ranks $S$, then $P$ is $H C M$ (resp. CM).

Since Walker's theorem is unpublished we give in Appendix I a version of his proof which is distilled from his letter [20].

One might ask whether Walker's theorem extends to the nonpure setting, namely if $P$ is semipure and $P_{S}$ is sequentially acyclic for all sets of rank levels $S$, then is $P$ is $S C M$. However, this is false, as shown by the following counterexample.

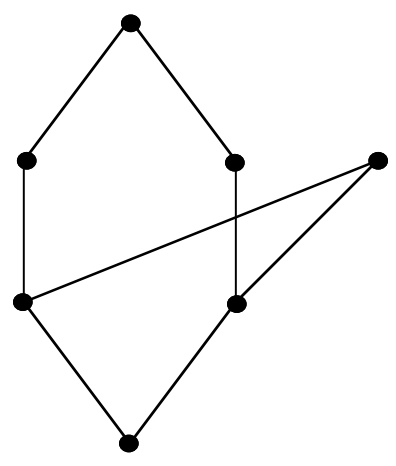


By combining Walker's theorem and Theorem 5.1 one gets a somewhat weaker extension of Walker's theorem, which is included in the following list of characterizations of $\mathrm{S}(\mathrm{H})$ CMness.

Corollary 5.3. Let $P$ be a semipure poset. Then the following are equivalent.

(1) $P$ is $S C M$ (resp. SHCM).

(2) $\left(P^{[j]}\right)_{S}$ is $(|S|-1)$-acyclic (resp. $(|S|-1)$-connected) for all $j$ and $S$ such that $S \subseteq[j]$.

(3) $\left(P^{[j]}\right)_{S}$ is $(|S|-1)$-acyclic (resp. $(|S|-1)$-connected) for all $j$ and every interval of ranks $S$ such that $S \subseteq[j]$.

(4) $\left(P^{<j>}\right)_{S}$ is $(|S|-1)$-acyclic (resp. $(|S|-1)$-connected) for all $j$ and $S$ such that $S \subseteq[j]$.

(5) $\left(P^{<j>}\right)_{S}$ is $(|S|-1)$-acyclic (resp. $(|S|-1)$-connected) for all $j$ and every interval of ranks $S$ such that $S \subseteq[j]$.

Proof. $(1) \Rightarrow(2)$. Use Theorem 5.1 and Corollary 4.4.

$(2) \Rightarrow(3)$. Obvious.

$(3) \Rightarrow(1)$. Use Theorems 5.2 and 5.1 .

$(2) \Longleftrightarrow(4)$ and $(3) \Longleftrightarrow(5)$. Use the fact that $\left(P^{[j]}\right)_{S}=\left(P^{<j>}\right)_{S}$ whenever $S \subseteq[j]$.

\section{Appendix I: Walker's Proof}

Proof of Theorem 5.2. We prove only the homotopy version, leaving the parallel reasoning in the homology case to the reader. The argument proceeds in three stages, making use of the following three properties of topological connectivity:

(i) If $X$ is an $n$-connected complex and $Y$ is an $(n-1)$-connected subcomplex, then $X / Y$ is $n$-connected.

(ii) Let $X=\bigvee X_{i}$ be a wedge of complexes. Then $X$ is $n$-connected if and only if each $X_{i}$ is $n$-connected.

(iii) The suspension $\operatorname{susp}(X)$ is $n$-connected if and only if $X$ is $(n-$ 1)-connected.

Step 1: If $P$ is a pure poset such that $P_{S}$ is spherical for every lower interval of ranks $S$, and $a \in P$, then $P_{<a}$ is spherical.

We may assume that $a$ is maximal in $P$. Let $n+1$ be the dimension of $X:=\Delta(P)$, and let $Y$ be the rank-selected subposet of $P$ formed by deleting the level $P_{\max }$ containing $a$. Then $X$ is $n$-connected and $Y$ is $(n-1)$-connnected, so $X / Y$ is $n$-connected. Since

$$
X / Y \cong \bigvee_{i \in P_{\max }} \operatorname{susp}\left(P_{<i}\right)
$$


it follows that $\operatorname{susp}\left(P_{<i}\right)$ is $n$-connected for all $i \in P_{\max }$. In particular, $P_{<a}$ is $(n-1)$-connected.

Step 2: If $P$ is a pure poset such that $P_{S}$ is spherical for every interval of ranks $S$, and $a \in P$, then $(P<a)_{D}$ is spherical for every upper interval $D$ of ranks of $P_{<a}$.

Suppose $D$ is an upper interval of ranks for $P_{<a}$, and let $\ell$ be the index of the level of $P$ which contains $a$. Note that

$$
\left(P_{<a}\right)_{D}=\left(P_{D \cup\{\ell\}}\right)_{<a} .
$$

The rank-selected subposet $P_{D \cup\{\ell\}}$ has the property that its rankselected subposet for every interval of ranks is spherical, so $\left(P_{D \cup\{\ell\}}\right)_{<a}$ is spherical by Step 1.

Step 3: We complete the proof.

If $b<a$ are elements of $P$, we want to show that the open interval $(b, a)$ is spherical. Observe that $(b, a)=\left(P_{<a}\right)_{>b}$. By Step 2, $P_{<a}$ is a ranked poset such that every upper rank-selected subposet is spherical. Now apply the dual of Step 1.

\section{Appendix II: Sequential Cohen-Macaulayness in Commutative Algebra}

For a simplicial complex $\Delta$ over ground set $\{1, \ldots, n\}$, we denote by $\mathbf{k}[\Delta]$ its Stanley-Reisner ring; that is the quotient of $S=\mathbf{k}\left[x_{1}, \ldots, x_{n}\right]$ by the ideal $I_{\Delta}$ generated by the monomials $x_{A}=\prod_{i \in A} x_{i}$ in $S$ for $A \notin \Delta$. A simplicial complex $\Delta$ was defined by Stanley [17] to be sequentially Cohen-Macaulay over a field $\mathbf{k}$ if $\mathbf{k}[\Delta]$ is a sequentially Cohen-Macaulay S-module. Again Stanley [17, Definition 2.9] defines a graded module $M$ over a standard graded k-algebra $R$ to be sequentially Cohen-Macaulay, if there is a filtration of submodules

$$
0=M_{0} \subset M_{1} \subset \cdots \subset M_{s}=M
$$

for which

$\triangleright M_{i} / M_{i-1}$ is a Cohen-Macaulay $R$-module.

$\triangleright \mathfrak{d i m} M_{1} / M_{0}<\cdots<\mathfrak{d i m} M_{s} / M_{s-1}$, where $\mathfrak{d i m}$ denotes Krull dimension.

The same concept appears in the work of Schenzel [16] under the name Cohen-Macaulay filtered module. Stanley mentions, and Schenzel verifies [16, Proposition 4.3], that if such a filtration exists then it is unique. From [16, Proposition 4.3] it also follows that such a filtration must coincide with the filtration defined as follows. Let $N_{j}$ be the maximal submodule of $M$ of dimension $\leq j$, then $N_{-1}=0 \subseteq N_{0} \subseteq \cdots \subseteq$ $N_{\mathfrak{d i m} M}=M$. If $M$ is sequentially Cohen-Macaulay [16, Proposition 
4.3] implies that for the indices $-1=j_{0}<\cdots<j_{s}=\mathfrak{d i m} M$ for which $N_{j_{i}-1} \neq N_{j_{i}}, i \geq 1$, we have $M_{i}=N_{j_{i}}, i \geq 0$.

In order to work out the maximal submodule of dimension $\leq j$ for a Stanley-Reisner ring $\mathbf{k}[\Delta]$ we define $\Delta_{j}$ as the subcomplex of $\Delta$ generated by its facets of dimension equal to $j$. (Recall that $\Delta^{\langle j\rangle}$ denotes the subcomplex of $\Delta$ generated by its facets of dimension $\geq j$ ). In general, if $\Gamma$ is a subcomplex of $\Delta$ then we denote by $I_{\Delta, \Gamma}$ the ideal generated in $\mathbf{k}[\Delta]$ by the monomials $x_{A}$ with $A \in \Delta \backslash \Gamma$. As an $S$ module $I_{\Delta, \Gamma}$ is isomorphic to $I_{\Gamma} / I_{\Delta}$. Now if $\Delta$ is a simplicial complex and if $j_{1}-1<\cdots<j_{s}-1$ are the dimensions of the facets of $\Delta$ then it follows that for $M=\mathbf{k}[\Delta]$ we have $M_{0}=0$ and

$$
M_{i-1}=I_{\Delta, \Delta\left\langle j_{i}-1\right\rangle}, i \geq 1 \text {. }
$$

By [17, III, Proposition 7.1]

$$
\mathfrak{d i m}\left(I_{\Delta, \Delta}\left\langle j_{i}-1\right\rangle\right)=j_{i-1} .
$$

One checks that $M_{i}$ is the maximal submodule of dimension $\leq j_{i}$ and that if $N$ is a maximal submodule of dimension $\leq d$, for some $d$, then $d=j_{i}$ for some $i$ and $N=M_{i}$.

Since

$$
I_{\Delta, \Delta\left\langle j_{i}-1\right\rangle} \cong I_{\Delta\left\langle j_{i}-1\right\rangle} / I_{\Delta}
$$

as $S$-modules, it follows that

$$
\begin{gathered}
M_{i-1} / M_{i-2} \cong I_{\Delta^{\left\langle j_{i}-1\right\rangle}} / I_{\Delta^{\left\langle j_{i-1}-1\right\rangle}} \cong \\
\cong I_{\Delta_{j_{i-1}-1}, \Delta_{j_{i-1}-1} \cap \Delta^{\left\langle j_{i}-1\right\rangle}} .
\end{gathered}
$$

The preceding isomorphism together with the definition of sequential Cohen-Macaulayness and the fact that

$$
\Delta^{\langle j\rangle}=\Delta_{j} \cup \cdots \cup \Delta_{\operatorname{dim}(\Delta)}
$$

then yield the following characterization of simplicial complexes $\Delta$ for which $\mathbf{k}[\Delta]$ is a sequential Cohen-Macaulay $S$-module, given in [17, III, Proposition 2.10]:

The $S$-module $\mathbf{k}[\Delta]$ is sequentially Cohen-Macaulay, if and only if

$$
I_{\Delta_{i}, \Delta_{i} \cap\left(\Delta_{i+1} \cup \cdots \cup \Delta_{\operatorname{dim}(\Delta)}\right)}
$$

is a Cohen-Macaulay module for all $i$.

The latter condition translates into $\left(\Delta_{i}, \Delta_{i} \cap\left(\Delta_{i+1} \cup \cdots \cup \Delta_{\operatorname{dim}(\Delta)}\right)\right)$ being a relative simplicial complex which is Cohen-Macaulay over $\mathbf{k}$. Stanley shows [17, III, Theorem 7.2] that a relative simplicial complex $(\Delta, \Gamma)$ is Cohen-Macaulay over $\mathbf{k}$ if and only if for all $A \in \Delta$ and all $i<\operatorname{dim}\left(\operatorname{lk}_{\Delta}(A)\right)$ we have $\widetilde{H}_{i}\left(\operatorname{lk}_{\Delta}(A), \operatorname{lk}_{\Gamma}(A) ; \mathbf{k}\right)=0$. This then 
gives rise to the characterizations of sequential Cohen-Macaulayness by Duval [10] (see Proposition 2.5) and Wachs [18] (see Definition 1.2 (i)). Our definition of sequentially homotopy Cohen-Macaulay is a natural homotopy version of this formulation.

In commutative algebra the concept of a sequentially Cohen-Macaulay module has been quite fruitful. We would like to mention only a few of the developments which also bear a combinatorial flavor.

- In [12] Herzog and Popescu characterize in ring-theoretic terms those sequentially Cohen-Macaulay $\mathbf{k}[\Delta]$ for which $\Delta$ is nonpure shellable. This generalizes the concept of cleanness by Dress [9] which characterizes the shellable $\Delta$ among the CohenMacaulay $\mathbf{k}[\Delta]$. Also in [12] a proof of a homological characterization of sequentially Cohen-Macaulay modules, originally due to Peskine [17], is given in terms of vanishing Ext-modules.

- It has been shown in [13] that $\mathbf{k}[\Delta]$ is sequentially CohenMacaulay if and only if $I_{\Delta^{*}}$ has a componentwise linear resolution, where $\Delta^{*}:=\{A \mid\{1, \ldots, n\} \backslash A \notin \Delta\}$ is the "combinatorial Alexander dual" of $\Delta$. This result generalizes a result by Eagon and Reiner [11] saying that $\mathbf{k}[\Delta]$ is Cohen-Macaulay if and only if $I_{\Delta^{*}}$ has a linear resolution. In [13] it is also shown that these facts imply that duals $\Delta^{*}$ of simplicial complexes $\Delta$ for which $\mathbf{k}[\Delta]$ is sequentially Cohen-Macaulay have the property that $\mathbf{k}\left[\Delta^{*}\right]$ is Golod. Despite substantial recent progress (see [2] and references therein) the latter property still waits for a satisfying combinatorial characterization.

- In [14] it is shown that for an ideal $I$ in a polynomial ring the Hilbert function of the local cohomology module of $R / I$ coincides with the one of $R / \operatorname{gin}(I)(\operatorname{gin}(I)$ being the generic initial ideal with respect to reverse lexicographic ordering) if and only if $R / I$ is sequentially Cohen-Macaulay.

\section{REFERENCES}

[1] K. Baclawski and A. M. Garsia, Combinatorial decompositions of a class of rings, Advances in Math. 39 (1981), 155-184.

[2] A. Berglund and M. Jöllenbeck, On the classification of the Golod property for Stanley-Reisner rings, J. Algebra, to appear.

[3] A. Björner, Topological Methods, Handbook of Combinatorics, R. Graham, M. Grötschel and L. Lovász, (Eds), North-Holland, Amsterdam, 1995, pp. 1819-1872.

[4] A. Björner and M.L. Wachs, Shellable nonpure complexes and posets, I, Trans. AMS 348 (1996), 1299-1327.

[5] A. Björner and M.L. Wachs, Nonpure shellable complexes and posets II, Trans. AMS 349 (1997), 3945-3975. 
[6] A. Björner and M.L. Wachs and V. Welker, Poset fiber theorems, Trans. Amer. Math. Soc. 357 (2005), 1877-1899.

[7] G.E. Bredon, Topology and Geometry, Graduate Texts in Mathematics, 139, Springer-Verlag, New York-Heidelberg-Berlin, 1993.

[8] W. Bruns and J. Herzog: Cohen-Macaulay Rings, Cambridge University Press, Cambridge, 1993.

[9] A. Dress, A new algebraic criterion for shellability, Beitr. Algebra Geom. 34, 45-55 (1993).

[10] A.M. Duval, Algebraic shifting and sequentially Cohen-Macaulay simplicial complexes, Electronic J. Combinatorics 3 (1996).

[11] J.A. Eagon and V. Reiner, Resolutions of Stanley-Reisner rings and Alexander duality, J. Pure Appl. Algebra 130 (1998), 265-275.

[12] J. Herzog and D. Popescu, Finite filtrations of modules and shellable multicomplexes, http://arxiv.org/abs/math.AC/0502282, Preprint 2005.

[13] J.Herzog and V. Reiner and V. Welker, Componentwise linear ideals and Golod rings, Mich. Math. J. 46 (1999), 211-223 (1999).

[14] J. Herzog and E. Sbarra, Sequentially Cohen-Macaulay modules and local cohomology, in Parimala, R. (ed.), Proceedings of the international colloquium on algebra, arithmetic and geometry, Tata Institute of Fundamental Research, Bombay. Stud. Math., Tata Inst. Fundam. Res. 16, 327-340 (2002).

[15] D. Quillen, Homotopy properties of the poset of non-trivial p-subgroups of a group, Advances in Math. 28 (1978), 101-128

[16] P. Schenzel, On the dimension filtration and Cohen-Macaulay filtered modules. In F. van Oystaeyen (ed.), Commutative algebra and algebraic geometry. New York, NY: Marcel Dekker. Lect. Notes Pure Appl. Math. 206, 245-264 (1999).

[17] R.P. Stanley, Combinatorics and Commutative Algebra, 2nd edition, Birkhäuser, Boston, 1995.

[18] M.L. Wachs, Whitney homology of semipure shellable posets, J. Algebraic Combinatorics 9 (1999), 173-207.

[19] M.L. Wachs, Poset topology: tools and applications, Geometric Combinatorics, IAS/Park City Math. Series, (Miller, Reiner, and Sturmfels, eds.), to appear.

[20] J.W. Walker, Letter to A. Björner dated October 20, 1981.

[21] J.W. Walker, Canonical homeomorphisms of posets, Europ. J. Combinatorics 9(1988), 97-107.

E-mail address: bjorner@math.kth.se

Department of Mathematics, Royal Institute of Technology, S10044 Stockholm, Sweden

E-mail address: wachs@math.miami.edu

Department of Mathematics, University of Miami, Coral Gables, FL 33124, USA

E-mail address: welker@mathematik.uni-marburg.de 
Fachbereich Mathematik und Informatik, Universität Marburg, D35032 Marburg, Germany 